\title{
Factores asociados con la actividad física en mujeres mexicanas en edad reproductiva
}

\author{
Bernardo Hernández, ${ }^{1}$ Jessica de Haene, ${ }^{2}$ Simón Barquera, ${ }^{2}$ \\ Eric Monterrubio, ${ }^{2}$ Juan Rivera, ${ }^{2}$ Teresa Shamah, ${ }^{2}$ \\ Jaime Sepúlveda, ${ }^{3}$ Jere Haas ${ }^{4}$ y Fabricio Campirano ${ }^{4}$
}

RESUMEN Objetivo. Documentar la práctica de actividades físicas ligeras y pesadas, particularmente de deportes y ejercicios, en mujeres de 12 a 49 años en México, y evaluar su asociación con algunas características sociodemográficas.

Métodos. Se estudió una muestra de 2367 mujeres de 12 a 49 años de edad residentes en México en 1999 mediante el análisis secundario de los datos de la Encuesta Nacional de Nutrición de México, 1999 (ENN-99). En esta encuesta se utilizó un esquema de muestreo polietápico, estratificado, por conglomerados que conformó una muestra final de 21000 hogares de todo el país. Mediante un cuestionario se evaluó el tiempo dedicado a las distintas formas de actividad física y se midió la actividad en equivalentes metabólicos (MET) según una escala puntual. Las variables dependientes del estudio fueron el tiempo dedicado a actividades físicas pesadas (las que exigen un gasto de energía equivalente a $5 \mathrm{MET} / \mathrm{hora}$ ) y el dedicado a actividades ligeras o sedentarias (tales como estar sentada en el trabajo o fuera de él, ver televisión, video, cine o teatro y dormir). Las variables independientes fueron la edad, escolaridad, paridad, región del país y área de residencia (urbana o rural), nivel socioeconómico (medido mediante un indice elaborado a partir de las condiciones de la vivienda y la posesión de enseres domésticos) y estado marital. Se evaluó la asociación entre las variables independientes y la práctica de deportes y se calcularon las razones de posibilidades brutas con intervalos de confianza de 95\%. Para identificar la asociación entre la práctica de deportes y las variables independientes se utilizó un modelo de regresión logística ajustado según el efecto de posibles variables de confusión. Se ajustó también mediante un factor de ponderación la probabilidad de que una mujer fuese seleccionada para la muestra, y para controlar el efecto de diseño producido por la agrupación de observaciones en esta muestra se utilizó la rutina de análisis de muestras complejas svy de Stata, versión 7.0.

Resultados. Se analizó la información obtenida de 2367 mujeres. Las mujeres encuestadas dedicaron 0,08 horas diarias, en promedio, a practicar deportes y 1,25 horas diarias a actividades físicas pesadas. Solo $16 \%$ informaron que practicaban algún deporte con regularidad. Al hacer ajustes según todas las variables independientes, las mujeres mayores de 30 años y las de 21 a 30 años tuvieron una menor razón de posibilidades $(R P)$ en lo que respecta a la práctica de deportes que las de 20 años o menos ( $R P=0,53$; intervalo de confianza de 95\% [IC95\%]: 0,33 a 0,84 y RP =0,35; IC95\%: 0,23 a 0,54, respectivamente). El mayor nivel de escolaridad y no haber tenido hijos fueron variables directamente asociadas con la práctica de

1 Instituto Nacional de Salud Pública, Centro de Investigación en Salud Poblacional, Cuernavaca, Morelos, México. La correspondencia debe ser enviada a Bernardo Hernández, Instituto Nacional de Salud Pública, Centro de Investigación en Salud Poblacional, Av. Universidad 655, Colonia
Santa María Ahuacatitlán, Cuernavaca, Morelos, México, 62508. Correo electrónico: bhernand@ insp.mx.

2 Instituto Nacional de Salud Pública, Centro de Investigación en Nutrición y Salud, Cuernavaca, México.
3 Instituto Nacional de Salud Pública, Dirección General, Cuernavaca, México.

4 Universidad de Cornell, Departamento de Nutrición, Ithaca, Nueva York, EUA. 
algún deporte. El área de residencia (urbana o rural), el estado marital y el nivel socioeconómico mostraron diferencias entre los grupos. No obstante, estas no resultaron significativas una vez que se hicieron los ajustes según las otras variables del estudio. El tiempo dedicado al deporte contribuyó solo en $10 \%$ al total de la actividad pesada y en $0,7 \%$ al total de la actividad física evaluada en MET. Las mujeres de 31 años de edad o más y las que tenían una escolaridad de nivel secundario tuvieron menos posibilidades de estar en el 10\% inferior en términos de actividad que las menores de 21 años o que las que tenían una escolaridad de nivel primario.

Conclusiones. Este estudio demuestra que la práctica de deportes no es frecuente como forma de actividad física en mujeres en edad reproductiva en México, especialmente entre las mayores de 20 años y las de baja escolaridad, lo que a su vez puede contribuir al aumento de la prevalencia de la obesidad y de las enfermedades crónicas.

Palabras clave Actividad física, deportes, mujeres, México.

México se encuentra en un proceso de transición en lo que respecta a la nutrición y la salud, en el transcurso del cual se ha experimentado un aumento sustancial de la incidencia de enfermedades crónicas no transmisibles. La tasa de mortalidad por estas enfermedades ha ascendido de 43,7 por 1000 habitantes en 1950 a 73 por 1000 habitantes en el año 2000 (1), y desde 1980 las enfermedades crónicas no transmisibles constituyen la principal causa de muerte en México (2). La mortalidad por diabetes mellitus, que representaba el $4 \%$ de la mortalidad general en 1955, abarcaba el 10\% en el año 2000 y ocupa desde 1999 el tercer lugar como causa de muerte, ocasionando la pérdida de 5,8\% del total de años de vida saludable en México (3). La mortalidad por enfermedades del corazón y el cáncer también ha mostrado aumentos notables en los últimos años $(2,4)$. Estas tendencias se observan en el grupo de mujeres en edad reproductiva, en el cual, además, la obesidad ha alcanzado niveles alarmantes. Se calcula que $30,6 \%$ de las mujeres mexicanas de 12 a 49 años tienen sobrepeso y que $21,2 \%$ de ellas presentan obesidad (5).

La actividad física, definida como todo movimiento corporal que implica un gasto de energía, es el componente del gasto energético que más varía y se encuentra, hasta cierto punto, bajo control voluntario (6). La actividad física desempeña un papel importante en la prevención de las enfermedades crónicas, pues se considera un factor de protección contra la cardiopatía isquémica, la diabetes mellitus, la enfermedad cerebrovascular, la osteoporosis, los trastornos mentales y algunos tipos de cáncer (7). Asimismo, se ha encontrado una asociación inversa entre la práctica de actividades físicas intensas y la obesidad (8).

Según datos de la Organización Mundial de la Salud, las enfermedades crónicas causan $60 \%$ de las muertes en el mundo, cifra que, según cálculos, podría ascender a 73\% para el año 2020, y gran parte de estas enfermedades están asociadas con el sedentarismo. En la Región de las Américas, la mayoría de la población realiza poca actividad física, cuya práctica disminuye con la edad. Las mujeres y las poblaciones de escasos recursos económicos tienden a ser menos activas físicamente que las de mayores recursos (9).

La práctica de actividades físicas está condicionada por factores determinados individualmente por cada persona, por la relación entre ellas y por el ambiente en que viven. Como problema de salud pública, la inactividad física es relativamente reciente, ya que es el desarrollo económico y social el que ha llevado a estilos de vida más sedentarios (10).

La importancia de la actividad física para la salud ha sido reconocida y se han hecho recomendaciones para lograr aumentar dicha actividad (11). No obstante, en México no se cuenta con información a escala nacional, ya sea acerca de la actividad física de toda la población o de la que corresponde específicamente a algún sector en particular, como el de las mujeres en edad reproductiva. La información existente se concentra en poblaciones jóvenes de ciudades específicas y apunta a que la actividad física es escasa (12). Es indispensable conocer los niveles de actividad física de las mujeres en edad reproductiva en México, así como los factores asociados con su práctica o ausencia, con el fin de diseñar intervenciones correctoras más eficaces y específicas.

El presente trabajo tiene como objetivo documentar la práctica de distintas formas de actividad física (ligera y pesada), particularmente la práctica de deportes y ejercicios, en mujeres de 12 a 49 años de edad en México, y evaluar su asociación con algunas características sociodemográficas.

\section{MATERIALES Y MÉTODOS}

La población estudiada se compuso de mujeres de 12 a 49 años de edad que residían en México en 1999. Se realizó un análisis secundario de los datos recolectados durante la Encuesta Nacional de Nutrición de México realizada en 1999 (ENN-99), en la que se empleó el Marco Muestral Nacional de Hogares, del Instituto Nacional de Estadística, Geografía e Informática (INEGI) (13), y se utilizó un esquema de muestreo probabilístico, polietápico, 
estratificado y por conglomerados. La ENN-99 tuvo representatividad nacional - tanto para estratos rurales como urbanos (localidades de más de 2500 habitantes) - y según las regiones del país (norte, centro, sur y Ciudad de México). La metodología de la ENN-99 ha sido descrita en detalle anteriormente (5).

La ENN-99 recogió información de 21000 hogares de toda la República Mexicana. El cuestionario fue aplicado a una submuestra compuesta por mujeres de 12 a 49 años. Para ello, entrevistadores previamente capacitados visitaron los hogares donde había mujeres comprendidas en este grupo de edad y recogieron información acerca de sus características sociodemográficas y actividad física.

El cuestionario sobre la actividad física, adaptación del propuesto por Bouchard y col. (14), constaba de 11 preguntas sobre el tiempo dedicado a las actividades físicas realizadas el día antes de la entrevista. Se diferenciaron las actividades físicas pesadas y las sedentarias. Las pesadas fueron definidas como aquellas que requieren un gasto de energía de $5 \mathrm{MET} /$ hora $^{5}$ o más, ya fueran realizadas en el ámbito laboral o fuera de él, así como la práctica de deportes y ejercicios. En cuanto a las actividades ligeras o sedentarias, se preguntó acerca del tiempo que las mujeres pasaban sentadas en el trabajo y fuera de él, el tiempo dedicado a ver televisión, video, cine o teatro, y el tiempo de sueño, incluida la siesta. Asimismo, se preguntó si el día al que se refería la entrevista había sido normal, más pesado o más ligero de lo usual, si las mujeres practicaban deportes habitualmente y con qué frecuencia. De esta forma fue posible calcular las horas diarias dedicadas a actividades pesadas (con especial hincapié en los deportes y los ejercicios, incluidos los practicados el día ante-

\footnotetext{
5 Un equivalente metabólico (MET) representa un múltiplo de la cantidad de oxígeno consumida en estado de reposo, la cual a su vez corresponde a 3,5 $\mathrm{mL} \mathrm{O} / \mathrm{kg} \cdot \mathrm{min}^{-1}$. Si al hacer cierto ejercicio una persona tiene un gasto energético de $10 \mathrm{MET}$, por ejemplo, significa que ha consumido 10 veces la cantidad de oxígeno que normalmente consumiría si estuviese en reposo.
}

rior a la encuesta y los que realizaban con regularidad) y a actividades ligeras en general, tanto en el ámbito laboral como fuera de él. Específicamente para la medición del tiempo dedicado a la práctica de deportes o ejercicios el día antes de la entrevista, se emplearon dos preguntas: "¿Ayer hizo algún deporte o ejercicio físico, como correr, andar en bicicleta, bailar, nadar, jugar voleibol o aeróbicos?" y, si la respuesta era positiva, se preguntaba además "¿Cuánto tiempo hizo deporte o ejercicios ayer?" Para medir la práctica habitual en materia de deportes, se preguntó "¿Practica regularmente algún deporte o ejercicio físico, como correr, andar en bicicleta, bailar, nadar, jugar voleibol o ejercicios aeróbicos?" Si la respuesta era positiva se preguntaba además "Por lo general, ¿cuántas horas a la semana (lunes a domingo) hace deporte o ejercicios?" El cuestionario no recogió información sobre actividades moderadas, por considerarse que el tiempo del día que no es dedicado a actividades ligeras ni pesadas les corresponde a estas otras.

Se debe aclarar que este cuestionario no fue diseñado para calcular exactamente el gasto de energía de las mujeres encuestadas, sino para identificar a las mujeres con mucha o con muy poca actividad física. Debido a que la información sobre el tiempo dedicado a las distintas formas de actividad física podía verse notablemente sesgada, las variables correspondientes se agruparon en dos categorías: las mujeres que practicaban deportes con regularidad y las que no los practicaban. En un estudio de validación realizado con mujeres de Morelos, México, se encontró que la práctica de deportes se correlacionaba de un modo significativo $(r=$ $0,22, P<0,05)$ con el consumo máximo de oxígeno, aunque la correlación con otras formas de actividad física no resultó significativa. ${ }^{6}$

A partir de la información sobre el tiempo dedicado a las distintas formas de actividad física el día antes de la entrevista se calcularon los equivalentes

\footnotetext{
6 De Haene J, Haas J, Hernández B, Rivera J, comunicación personal, 2002.
}

metabólicos, lo que sirvió para resumir la información de las actividades ligera y pesada. Esto permitió que no se analizaran los distintos tipos de actividad de manera aislada y clasificar a las mujeres encuestadas sobre una base biológica, de acuerdo con la actividad física total que realizaron el día antes de la entrevista. Los valores en MET utilizados originalmente para las distintas actividades del cuestionario fueron homologados según el Compendio de Actividades Físicas de Ainsworth (15).

Con el objetivo de evitar que se indicara un número excesivo de horas dedicadas a las diferentes actividades se realizaron ajustes según la plausibilidad de la información recogida de cada participante. Por ejemplo, la actividad física fue definida como ligera o sedentaria cuando requería un gasto de energía de 1,58 MET, pero este valor fue ajustado a 1,08 MET en el caso de mujeres que indicaron más de dos horas de este tipo de actividad. Para las actividades pesadas se utilizó un valor de 5 MET que fue ajustado a 4,0 MET en el caso de las mujeres que indicaron más de una hora de actividad pesada. Para la práctica de deportes el día anterior se definió un valor de 6,75 MET, el cual fue ajustado a 4,5 MET en el caso de las mujeres que indicaron haber realizado más de 30 minutos de ejercicio. Una vez que se obtuvieron el tiempo dedicado a cada forma de actividad física y su valor en MET, se calculó el valor total de MET por día. Tomando en cuenta que el tiempo de cada actividad física recogido en el cuestionario era solo aproximado y que se calculó a partir de la información sobre el día anterior a la entrevista, los valores de MET pueden tener algún grado de error. Para minimizarlo, las mujeres se clasificaron según estos valores en grupos de 10\% según su actividad y para el análisis se utilizaron solo el $10 \%$ de menor y el $10 \%$ de mayor actividad.

Entre las variables independientes se estudiaron algunas características que pudieran estar asociadas con la práctica de actividades físicas y deportes, tales como la edad, la residencia en áreas rurales o urbanas (poblaciones 
con más de 2500 habitantes) y la paridad. Las mujeres se clasificaron de acuerdo con cuatro regiones de residencia: norte (Baja California, Baja California Sur, Coahuila, Chihuahua, Durango, Nuevo León, Sonora y Tamaulipas), centro (Aguascalientes, Colima, Guanajuato, Jalisco, estado de México - excepto áreas conurbanas de la ciudad de México-, Michoacán, Morelos, Nayarit, Querétaro, San Luis Potosí, Sinaloa y Zacatecas), Ciudad de México (Distrito Federal y áreas conurbanas) y sur (Campeche, Chiapas, Guerrero, Hidalgo, Oaxaca, Puebla, Quintana Roo, Tabasco, Tlaxcala, Veracruz y Yucatán). Asimismo, se recogió información sobre el estado marital de las mujeres (no unidas: solteras, divorciadas, viudas y separadas; y unidas: casadas o en unión libre) y el nivel de escolaridad. El nivel socioeconómico se midió mediante un índice elaborado a partir de las condiciones de la vivienda (material del piso, disponibilidad de agua potable y drenaje) y la posesión de enseres domésticos (radio, televisión, refrigerador, lavadora de ropa y estufa), según el índice propuesto por Bronfman y colaboradores (16).

\section{Análisis}

Se realizó un análisis exploratorio de los datos con el fin de identificar las observaciones poco plausibles o extremas, describir las características de la población y calcular el tiempo dedicado a cada forma de actividad física. Se definieron como valores poco plausibles de actividad física aquellos cuya suma, referida al tiempo dedicado a las diferentes actividades en un solo día, daba un valor superior a 24 horas.

Para evaluar la asociación entre las variables independientes y la práctica de deportes se calcularon las razones de posibilidades (RP) brutas y sus intervalos de confianza de 95\%. Asimismo, se utilizó un modelo de regresión logística para identificar la asociación entre la práctica de deportes y las variables independientes, ajustando el efecto de posibles variables de confusión. Para estos análisis se utilizaron como variables dependientes la práctica de deportes el día antes de la encuesta y la práctica habitual de deportes, para toda la población de estudio y según el área de residencia (rural o urbana). Además, se ajustaron modelos de regresión logística para identificar las variables asociadas con la pertenencia de las mujeres al $10 \%$ de mayor o menor actividad física. Debido a que una proporción importante de mujeres aún podían estar asistiendo a la escuela hasta los 24 años de edad, también se ajustaron los modelos de regresión logística para evaluar el efecto de la educación únicamente entre mujeres mayores de 24 años.

Dado que en la ENN-99 se aplicó un muestreo polietápico por conglomerados, en todos los análisis se realizaron ajustes según la probabilidad de cada mujer de ser incluida en la muestra mediante el uso de un valor de ponderación. Asimismo, para controlar el efecto del diseño producido por la agrupación de observaciones en esta muestra se utilizó la rutina de análisis de muestras complejas soy de Stata, versión 7.0 (17). Finalmente, debido a que la prevalencia de las variables de interés principal (outcome variables) puede ser mayor de $10 \%$, se calcularon las razones de prevalencia de la práctica de los deportes y de la pertenencia al 10\% de mayor y de menor actividad física, de acuerdo con las variables independientes del estudio mediante el método propuesto por Zhang y Yu (18).

\section{RESULTADOS}

Durante la ENN-99 se recogió información sobre la actividad física de 2673 mujeres. De ellas se excluyó a 272 que no contestaron todas las preguntas o que proporcionaron información poco plausible. También se excluyó a 34 mujeres cuyos datos sociodemográficos estaban incompletos. De esta forma, la muestra final del estudio quedó integrada por 2367 mujeres. La mayor proporción de mujeres tenían más de 30 años (43,9\%), 63,6\% habitaban en áreas urbanas y 70,4\% estaban casadas o unidas maritalmente a una pareja. Con respecto a la escolaridad,
$53,5 \%$ de las encuestadas tenían estudios primarios, 24,2\% habían alcanzado la escuela secundaria y 22,3\% habían cursado estudios preparatorios o universitarios. Además, 27,1\% de las mujeres no habían tenido ningún parto, 50,0\% de ellas habían tenido de uno a tres partos, y 22,9\% habían tenido cuatro o más (cuadro 1 ).

De acuerdo con la información sobre el día anterior a la entrevista, las mujeres dedicaron 0,08 horas diarias, en promedio, a los ejercicios físicos -incluidos los deportes- y 1,25 horas diarias a actividades pesadas (incluidas las actividades laborales, las no laborales y los deportes). Las mujeres encuestadas indicaron que dedicaban como promedio 0,45 horas a la semana a practicar deportes o hacer ejercicios (cuadro 2). En general, 8,9\% de las mujeres indicaron haber dedicado como mínimo media hora a algún deporte el día antes de la entrevista y 15,9\% dijeron que practicaban algún deporte o ejercicio con regularidad (figura 1).

Se analizó la asociación utilizando la práctica de un deporte el día anterior a la entrevista y la práctica habitual de algún deporte como variables dependientes, y las características sociodemográficas de las mujeres como variables independientes. Debido a que los resultados con ambas variables dependientes fueron similares, en este artículo se presentan únicamente los resultados utilizando la práctica habitual de deportes como variable dependiente. Las posibilidades de practicar deportes fueron significativamente menores entre las mujeres mayores de 21 años que entre las más jóvenes (cuadro 3). Al ajustar estos resultados según las variables independientes del estudio (región del país y área de residencia, estado marital, escolaridad, nivel socioeconómico y paridad), esta asociación se mantuvo y las mujeres mayores de 30 años y las de 21 a 30 años tuvieron una menor razón de posibilidades de practicar deporte que las de 20 años o menos (RP = 0,53; IC95\%: 0,33 a 0,84 y $R P=0,35$; IC95\%: 0,23 a 0,54 , respectivamente). Las mujeres que residían en áreas urbanas también tuvieron mayores posibilidades de practicar deportes $(\mathrm{RP}=1,39$; IC $95 \%$ : 
CUADRO 1. Características sociodemográficas de las mujeres estudiadas. Encuesta Nacional de Nutrición, México, 1999 ( $n=2$ 367)

\begin{tabular}{|c|c|c|}
\hline Característica & No. & $\%$ \\
\hline $\begin{array}{l}\text { Grupo de edad } \\
\geq 31 \text { años } \\
\text { De } 21 \text { a } 30 \text { años } \\
\leq 20 \text { años }\end{array}$ & $\begin{array}{r}1040 \\
828 \\
499\end{array}$ & $\begin{array}{l}43,9 \\
35,0 \\
21,1\end{array}$ \\
\hline $\begin{array}{l}\text { Área de residencia } \\
\text { Urbana (> } 2500 \text { habitantes) } \\
\text { Rural }\end{array}$ & $\begin{array}{r}1505 \\
862\end{array}$ & $\begin{array}{l}63,6 \\
36,4\end{array}$ \\
\hline $\begin{array}{l}\text { Región } \\
\text { Norte } \\
\text { Centro } \\
\text { Ciudad de México } \\
\text { Sur }\end{array}$ & $\begin{array}{l}705 \\
663 \\
252 \\
747\end{array}$ & $\begin{array}{l}29,8 \\
28,0 \\
10,6 \\
31,6\end{array}$ \\
\hline $\begin{array}{l}\text { Estado marital } \\
\text { No unida (soltera, divorciada, viuda, separada) } \\
\text { Unida (casadas o en unión libre) }\end{array}$ & $\begin{array}{r}701 \\
1666\end{array}$ & $\begin{array}{l}29,6 \\
70,4\end{array}$ \\
\hline $\begin{array}{l}\text { Nivel de escolaridad } \\
\text { Preparatoria o más } \\
\text { Secundaria } \\
\text { Primaria }\end{array}$ & $\begin{array}{r}528 \\
573 \\
1266\end{array}$ & $\begin{array}{l}22,3 \\
24,2 \\
53,5\end{array}$ \\
\hline $\begin{array}{l}\text { Nivel socioeconómico a } \\
\text { Alto } \\
\text { Medio } \\
\text { Bajo }\end{array}$ & $\begin{array}{l}815 \\
765 \\
787\end{array}$ & $\begin{array}{l}34,4 \\
32,3 \\
33,3\end{array}$ \\
\hline $\begin{array}{l}\text { Paridad } \\
4 \text { ó más } \\
\text { De } 1 \text { a } 3 \\
0\end{array}$ & $\begin{array}{r}542 \\
1184 \\
641\end{array}$ & $\begin{array}{l}22,9 \\
50,0 \\
27,1\end{array}$ \\
\hline
\end{tabular}

${ }^{a}$ Construido a partir de un índice que toma en cuenta las condiciones de la vivienda y la posesión de enseres domésticos.

CUADRO 2. Medias, medianas y desviaciones estándar del tiempo (horas diarias) dedicado a las distintas formas de actividad por las mujeres estudiadas. Encuesta Nacional de Nutrición, México, $1999(n=2367)^{\mathrm{a}}$

\begin{tabular}{lccc}
\hline \multicolumn{1}{c}{ Tipo de actividad ${ }^{\mathrm{b}}$} & Mediac $^{\mathrm{c}}$ & Mediana $^{\mathrm{c}}$ & $\begin{array}{c}\text { Desviación } \\
\text { estándarc }^{\mathrm{c}}\end{array}$ \\
\hline Actividad laboral pesada & 0,12 & 0 & 0,96 \\
Actividad pesada no laboral (excepto deportes) & 1,05 & 0,5 & 1,63 \\
Deportes o ejercicios el día anterior & 0,08 & 0 & 0,27 \\
Total de actividad pesada & 1,25 & 0,5 & 1,95 \\
Actividad laboral ligera & 0,19 & 0 & 1,13 \\
Actividad ligera no laboral & 3,64 & 3,5 & 2,67 \\
Televisión, cine o teatro & 1,15 & 1,5 & 1,22 \\
Sueño nocturno & 8,10 & 7 & 1,91 \\
Siesta & 0,25 & 0 & 0,65 \\
Total de horas de sueño & 8,35 & 9,5 & 1,95 \\
Total de actividad ligera & 13,39 & 13 & 3,62 \\
Deportes o ejercicios habituales & 0,45 & 0 & 1,15 \\
\hline a La información se expresa en horas diarias dedicadas a cada forma de actividad y se refiere al día anterior a la entrevista, \\
con la excepción de "Deportes o ejercicios habituales", que se refiere a la práctica habitual. \\
b Actividad pesada es aquella que requiere un gasto de energía equivalente a cinco múltiplos del metabolismo basal/hora \\
(MET/hora). El total de actividad pesada incluye la actividad pesada realizada en el ámbito laboral y fuera de él (excepto de- \\
portes), y los deportes y ejercicios realizados el día anterior a la encuesta. La actividad ligera o sedentaria incluye el tiempo \\
dedicado a estar sentada, ver televisión, video, y cine o teatro. El total de horas de sueño incluye las horas de sueño noc- \\
turno y de siesta. El total de actividad ligera incluye la actividad ligera realizada en el ámbito laboral y fuera de él, el tiempo \\
dedicado a ver televisión, cine y teatro, y el total de horas de sueño.
\end{tabular}

0,99 a 1,96); no obstante, esa asociación no resultó significativa una vez que se hicieron ajustes según las otras variables del estudio. Las mujeres sin unión marital tuvieron 2,62 veces más posibilidades de practicar deportes que las casadas y las unidas maritalmente a una pareja $(\mathrm{RP}=2,62$; IC95\%: 1,98 a $3,47)$, aunque esta asociación también perdió su significación estadística cuando se aplicó el modelo de múltiples variables. No se encontraron diferencias en la práctica de deportes entre las mujeres de las diferentes regiones del país.

El nivel de escolaridad mostró una asociación directa con la práctica de deportes, tanto en los modelos brutos como en el modelo de múltiples variables. Al ajustar los resultados según las otras variables del estudio, las mujeres con escolaridad de secundaria y preparatoria o más tuvieron mayores posibilidades de practicar deportes que las que apenas tenían una educación primaria. Se encontraron resultados similares al hacer este análisis únicamente con la información de las mujeres mayores de 24 años, es decir, la asistencia a alguna institución estudiantil en el momento de la investigación no influyó en estos resultados. El nivel socioeconómico también estuvo directamente asociado con la práctica de deportes en el análisis bruto, pero no después de ajustar los resultados según las otras variables. Finalmente, la paridad y la práctica de deportes mantuvieron una asociación inversa. Después de ajustar según las otras variables, se observó que las mujeres que habían parido tenían menores posibilidades de practicar deportes que las que no habían tenido hijos. Las razones de prevalencia calculadas a partir de las razones de posibilidades del modelo de múltiples variables fueron muy similares a estas (cuadro 3).

La asociación entre las variables estudiadas y la práctica de deportes entre las mujeres de áreas urbanas y rurales fue similar a la encontrada en la población de estudio en su conjunto. Sin embargo, la asociación observada entre la práctica de los deportes y la escolaridad fue significativa, tanto entre las mujeres que vivían en áreas rurales 
FIGURA 1. Proporción de mujeres que realizan distintas formas de actividad física pesada ( $n=2$ 367). Encuesta Nacional de Nutrición, México, 1999

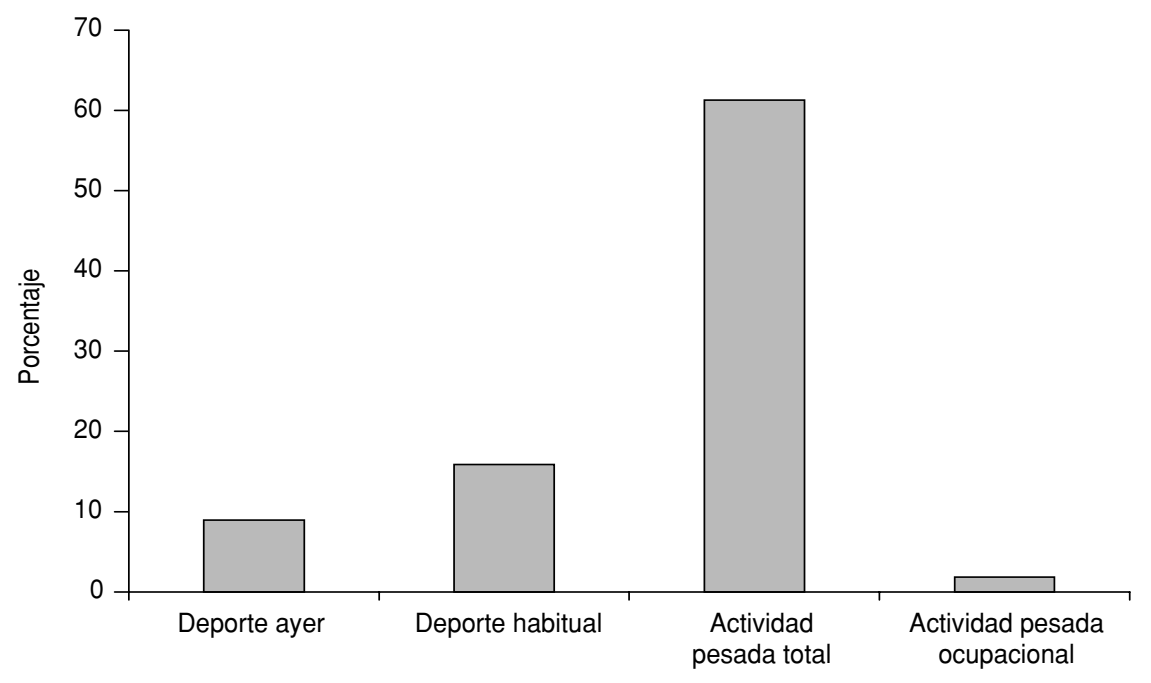

Nota: La actividad pesada es aquella que requiere un gasto de energía equivalente a cinco múltiplos del metabolismo basal/hora (MET/hora). La actividad pesada total incluye la actividad pesada realizada en el ámbito laboral y fuera de él (excepto deportes) y los deportes y ejercicios realizados el día anterior.

CUADRO 3. Razones de posibilidades brutas y ajustadas según las características sociodemográficas de las mujeres. Encuesta Nacional de Nutrición, México, 1999 ( $n=2$ 367)

\begin{tabular}{|c|c|c|c|c|}
\hline Característica & $\begin{array}{l}\text { Mujeres que } \\
\text { practican } \\
\text { deportes, \% }\end{array}$ & 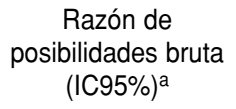 & $\begin{array}{c}\text { Razón de } \\
\text { posibilidades } \\
\text { ajustadab (IC95\%) }\end{array}$ & $\begin{array}{l}\text { Razón de } \\
\text { prevalencias } \\
\text { (IC95\%) }\end{array}$ \\
\hline \multicolumn{5}{|l|}{ Grupo de edad } \\
\hline$\geq 31$ años & 10,7 & $0,30(0,22$ a 0,42$)$ & $0,53(0,33$ a 0,84$)$ & $0,60(0,40$ a 0,87$)$ \\
\hline De 21 a 30 años & 12,1 & $0,27(0,19$ a 0,39$)$ & $0,35(0,23$ a 0,54$)$ & $0,42(0,28$ a 0,61$)$ \\
\hline$\leq 20$ años & 33,3 & 1 & 1 & \\
\hline \multicolumn{5}{|l|}{ Área de residencia } \\
\hline Urbana (> 2500 habitantes) & 18,1 & $1,39(0,99$ a 1,96$)$ & $1,08(0,71$ a 1,65$)$ & $1,07(0,74$ a 1,53$)$ \\
\hline Rural & 12,1 & 1 & 1 & \\
\hline \multicolumn{5}{|l|}{ Región } \\
\hline Norte & 13,8 & $0,93(0,64$ a 1,33$)$ & $0,83(0,55$ a 1,24$)$ & $0,85(0,59$ a 1,20$)$ \\
\hline Centro & 15,8 & $0,86(0,60$ a 1,25$)$ & $0,92(0,62$ a 1,38$)$ & $0,93(0,65$ a 1,32$)$ \\
\hline Ciudad de México & 19,8 & $1,11(0,72$ a 1,71$)$ & $0,92(0,56$ a 1,51$)$ & $0,93(0,59$ a 1,41$)$ \\
\hline Sur & 16,7 & 1 & 1 & \\
\hline \multicolumn{5}{|l|}{ Estado marital } \\
\hline No unida (soltera, divorciada, viuda, separada) & 27,2 & $2,62(1,98$ a 3,47$)$ & $1,23(0,86$ a 1,77$)$ & $1,18(0,88$ a 1,55$)$ \\
\hline Unida (casadas o en unión libre) & 11,2 & 1 & 1 & \\
\hline \multicolumn{5}{|l|}{ Nivel de escolaridad } \\
\hline Preparatoria o más & 26,7 & $2,86(2,01$ a 4,05$)$ & $2,62(1,66$ a 4,12$)$ & $2,31(1,58$ a 3,27$)$ \\
\hline Secundaria & 20,6 & $2,55(1,79$ a 3,62$)$ & $2,00(1,32$ a 3,04$)$ & $1,85(1,29$ a 2,60$)$ \\
\hline Primaria & 9,3 & 1 & & \\
\hline \multicolumn{5}{|l|}{ Nivel socioeconómico ${ }^{d}$} \\
\hline Alto & 22,7 & $1,82(1,29$ a 2,55$)$ & $1,33(0,83$ a 2,13$)$ & $1,28(0,85$ a 1,87$)$ \\
\hline Medio & 13,5 & $1,27(0,87$ a 1,85$)$ & $1,10(0,71$ a 1,69$)$ & $1,08(0,74$ a 1,56$)$ \\
\hline Bajo & 11,3 & 1 & 1 & \\
\hline \multicolumn{5}{|l|}{ Paridad } \\
\hline 4 ó más & 6,9 & $0,18(0,12$ a 0,28$)$ & $0,43(0,24$ a 0,78$)$ & $0,48(0,27$ a 0,81$)$ \\
\hline De 1 a 3 & 12,8 & $0,36(0,26$ a 0,49$)$ & $0,62(0,40$ a 0,95$)$ & $0,66(0,45$ a 0,96$)$ \\
\hline 0 & 28,9 & 1 & 1 & \\
\hline
\end{tabular}

a Ajustada según las variables incluidas en el cuadro y calculada mediante ponderadores muestrales.

b IC95\%: intervalo de confianza de $95 \%$.

c Calculada por el método propuesto por Zhang y Yu (17) a partir de las razones de posibilidades ajustadas.

${ }^{\mathrm{d}}$ Construido a partir de un índice basado en las condiciones de la vivienda y la posesión de enseres domésticos. 
CUADRO 4. Razones de posibilidades ajustadas de la práctica habitual de deportes según las características sociodemográficas de las mujeres de áreas urbanas y rurales. Encuesta Nacional de Nutrición, México, 1999 ( $n=2367)$

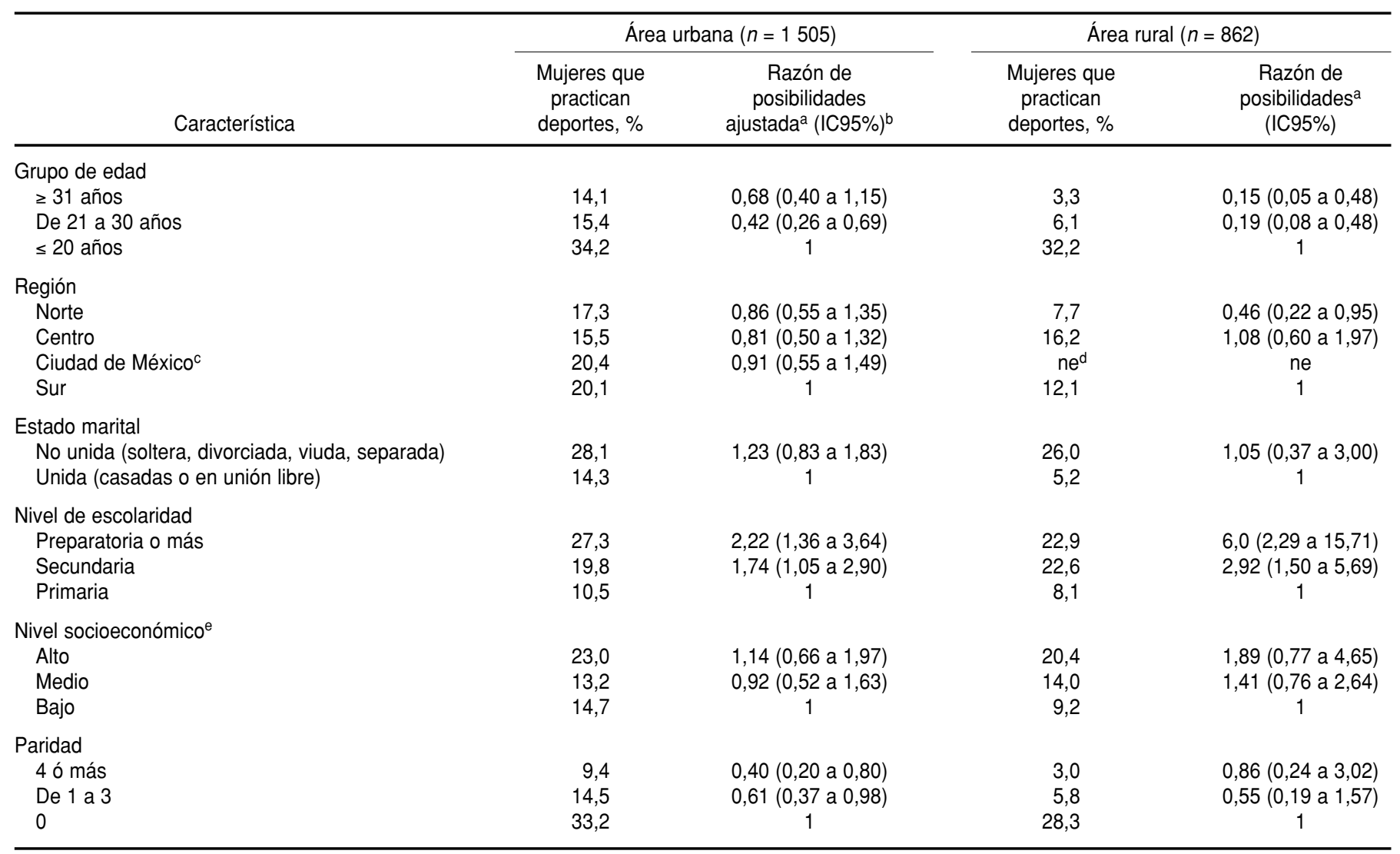

a Ajustada según las variables incluidas en el cuadro y calculada mediante ponderadores muestrales.

b IC95\%: intervalo de confianza de $95 \%$.

${ }^{c}$ No se incluyen datos del área rural de la Ciudad de México dado el número reducido de observaciones $(n=12)$

${ }^{\mathrm{d}} \mathrm{Ne}$ : no evaluado.

e Construido a partir de un índice basado en las condiciones de la vivienda y la posesión de enseres domésticos.

como entre las que vivían en áreas urbanas (cuadro 4).

Según la puntuación en MET, utilizada como medida de la actividad física practicada, el tiempo dedicado al deporte contribuyó solo en $10 \%$ al total de actividades pesadas y en $0,7 \%$ a la actividad física total (figura 2). Al clasificar a las mujeres en grupos de $10 \%$ de acuerdo con su actividad física, se encontró que después de hacer los ajustes según las variables estudiadas (edad, región del país y área de residencia, estado marital, escolaridad, nivel socioeconómico y paridad), las mujeres de 31 años o más tenían una menor probabilidad de estar en el 10\% de menor actividad que las de 20 años o menos, a pesar de las diferencias encontradas en cuanto a la práctica de deportes. Asi-
FIGURA 2. Distribución del gasto total de energía por tipo de actividad en las mujeres estudiadas. Encuesta Nacional de Nutrición, México, 1999
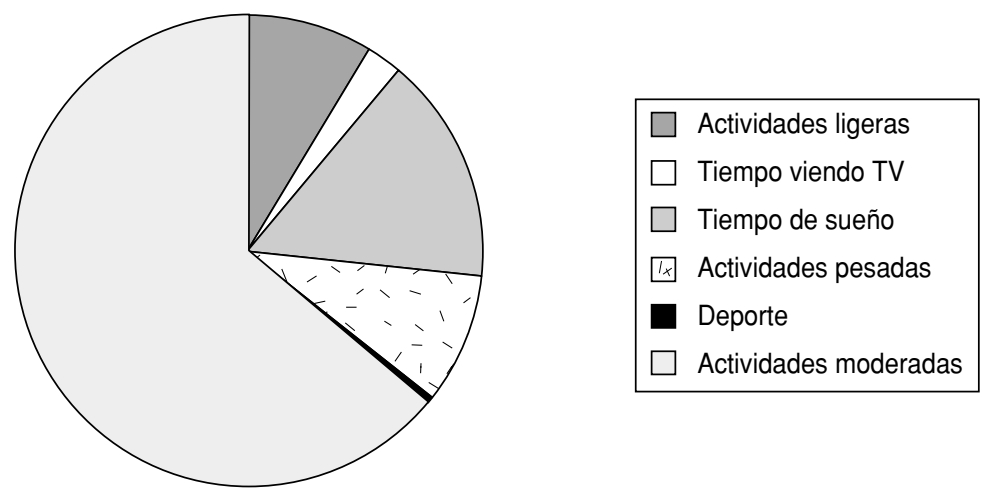

Nota: La actividad pesada es aquella que requiere un gasto de energía equivalente a 5 veces el valor del metabolismo basal/hora (MET/hora). El total de horas de sueño incluye las horas de sueño nocturno y de siesta. El total de actividad ligera incluye la actividad ligera realizada en el ámbito laboral y fuera de él, el tiempo dedicado a ver televisión, cine y teatro, y el total de horas de sueño. 
CUADRO 5. Razones de posibilidades brutas y ajustadas de mujeres en edad reproductiva en el $10 \%$ de menor actividad física ${ }^{a}$. Encuesta Nacional de Nutrición, México, 1999

\begin{tabular}{|c|c|c|c|}
\hline Característica & $\begin{array}{l}\text { Mujeres con poca } \\
\text { actividad física, \% }\end{array}$ & $\begin{array}{l}\text { Razón de posibilidades } \\
\text { bruta (IC95\%) }\end{array}$ & $\begin{array}{l}\text { Razón de posibilidades } \\
\text { ajustada }^{\mathrm{C}}(\mathrm{IC} 95 \%)\end{array}$ \\
\hline \multicolumn{4}{|l|}{ Grupo de edad } \\
\hline$\geq 31$ años & 5,7 & $0,33(0,21$ a 0,52$)$ & $0,33(0,17$ a 0,66$)$ \\
\hline De 21 a 30 años & 11,2 & $0,70(0,45$ a 1,08$)$ & $0,64(0,36$ a 1,12$)$ \\
\hline$\leq 20$ años & 16,8 & 1 & 1 \\
\hline \multicolumn{4}{|l|}{ Área de residencia } \\
\hline Urbana (> 2500 habitantes) & 11,1 & $1,75(1,21$ a 2,52$)$ & $1,49(0,93$ a 2,38$)$ \\
\hline Rural & 8,0 & 1 & 1 \\
\hline \multicolumn{4}{|l|}{ Región } \\
\hline Norte & 13,2 & $1,62(1,05$ a 2,51$)$ & $1,48(0,91$ a 2,40$)$ \\
\hline Centro & 8,6 & $1,07(0,67$ a 1,71$)$ & $1,03(0,64$ a 1,66$)$ \\
\hline Ciudad de México & 10,7 & $1,32(0,75$ a 2,34$)$ & $1,02(0,58$ a 1,82) \\
\hline Sur & 7,9 & 1 & 1 \\
\hline \multicolumn{4}{|l|}{ Estado marital } \\
\hline No unida (soltera, divorciada, viuda, separada) & 14,5 & $1,64(1,14$ a 2,36$)$ & $1,01(0,58$ a 1,75$)$ \\
\hline Unida (casadas o en unión libre) & 8,0 & 1 & 1 \\
\hline \multicolumn{4}{|l|}{ Nivel de escolaridad } \\
\hline Preparatoria o más & 14,6 & $2,01(1,32$ a 3,05$)$ & $1,40(0,85$ a 2,33$)$ \\
\hline Secundaria & 11,3 & $1,04(0,66$ a 1,63$)$ & $0,61(0,39$ a 0,99$)$ \\
\hline Primaria & 7,4 & 1 & 1 \\
\hline \multicolumn{4}{|l|}{ Nivel socioeconómico ${ }^{d}$} \\
\hline Alto & 13,0 & $1,90(1,20$ a 3,00$)$ & $1,45(0,80$ a 2,63$)$ \\
\hline Medio & 10,1 & $1,77(1,06$ a 2,94$)$ & $1,56(0,91$ a 2,68$)$ \\
\hline Bajo & 6,7 & 1 & 1 \\
\hline \multicolumn{4}{|l|}{ Paridad } \\
\hline 4 ó más & 3,5 & $0,20(0,10$ a 0,38$)$ & $0,42(0,17$ a 1,04$)$ \\
\hline De 1 a 3 & 9,7 & $0,65(0,44$ a 0,96$)$ & $0,92(0,49$ a 1,72$)$ \\
\hline 0 & 15,4 & 1 & 1 \\
\hline
\end{tabular}

mismo, las mujeres con escolaridad de nivel secundario tuvieron menos posibilidades de estar en el $10 \%$ de actividad más bajo que las que tenían una escolaridad de nivel primario (cuadro 5). El resto de las variables estudiadas no mostraron diferencias significativas.

Las razones de posibilidades brutas y ajustadas de estar en el 10\% de mayor actividad física de acuerdo con las variables independientes del estudio se presentan en el cuadro 6. Una vez que se hicieron los ajustes según las variables independientes del estudio, las mujeres mayores de 31 años tuvieron mayores posibilidades de estar en el $10 \%$ de mayor actividad física que las mujeres de 20 años o menos, mientras que las mujeres de nivel socioeconómico medio tuvieron menores posibilidades de estar en el $10 \%$ de mayor actividad comparadas con las mujeres de nivel socioeconómico bajo. El resto de las variables estudiadas no se mostraron asociadas de un modo significativo con la ubicación de las mujeres en el 10\% de mayor actividad, según el modelo de múltiples variables (cuadro 6).

\section{DISCUSIÓN}

Los resultados de este estudio muestran que las mujeres en edad reproductiva en México practican poco deporte. Este es el primer estudio que documenta esta situación en una muestra probabilística a escala nacional. Además, indica que la práctica de los deportes es menos frecuente entre las mujeres mayores de 21 años y más frecuente entre las de mayor escolaridad.

La práctica menos frecuente de los deportes entre las mujeres mayores de 30 años encontrada en este estudio coincide con lo observado por otros autores. Se ha documentado que la práctica de actividad física en general disminuye con la edad, especialmente durante la transición a la adolescencia (19). Los resultados de la presente investigación indican que este patrón puede continuar durante la adultez. No obstante, es posible que la escasa actividad física de las mujeres mayores se deba al efecto de cohorte (si las 
CUADRO 6. Razones de posibilidades brutas y ajustadas de mujeres en edad reproductiva con mucha actividad física. ${ }^{a}$ Encuesta Nacional de Nutrición, México 1999

\begin{tabular}{|c|c|c|c|}
\hline Característica & $\begin{array}{l}\text { Mujeres con poca } \\
\text { actividad física, \% }\end{array}$ & $\begin{array}{l}\text { Razón de posibilidades } \\
\text { bruta (IC95\%) }\end{array}$ & $\begin{array}{l}\text { Razón de posibilidades } \\
\text { ajustadac (IC95\%) }\end{array}$ \\
\hline \multicolumn{4}{|l|}{ Grupo de edad } \\
\hline$\geq 31$ años & 14,5 & $3,34(2,08$ a 5,38$)$ & $2,65(1,31$ a 5,34$)$ \\
\hline De 21 a 30 años & 8,3 & $1,85(1,05$ a 3,26$)$ & $1,61(0,71$ a 3,66$)$ \\
\hline$\leq 20$ años & 6,0 & 1 & 1 \\
\hline \multicolumn{4}{|l|}{ Área de residencia } \\
\hline Urbana (> 2500 habitantes) & 9,8 & $0,76(0,54$ a 1,06$)$ & $0,84(0,58$ a 1,21$)$ \\
\hline Rural & 11,8 & 1 & 1 \\
\hline \multicolumn{4}{|l|}{ Región } \\
\hline Norte & 7,9 & $0,68(0,43$ a 1,07$)$ & $0,80(0,50$ a 1,26$)$ \\
\hline Centro & 10,3 & $0,87(0,58$ a 1,32$)$ & $0,94(0,63$ a 1,42$)$ \\
\hline Ciudad de México & 11,1 & $1,09(0,62$ a 1,91$)$ & $1,50(0,84$ a 2,67$)$ \\
\hline Sur & 13,1 & 1 & 1 \\
\hline \multicolumn{4}{|l|}{ Estado marital } \\
\hline No unidas (soltera, divorciada, viuda, separada) & 7,7 & $0,60(0,40$ a 0,89$)$ & $1,08(0,61$ a 1,94$)$ \\
\hline Unidas (casadas o en unión libre) & 11,8 & 1 & 1 \\
\hline \multicolumn{4}{|l|}{ Nivel de escolaridad } \\
\hline Preparatoria o más & 7,9 & $0,76(0,38$ a 0,96$)$ & $0,73(0,42$ a 1,28$)$ \\
\hline Secundaria & 8,9 & $0,60(0,46$ a 1,23$)$ & $1,13(0,69$ a 1,84$)$ \\
\hline Primaria & 12,4 & 1 & 1 \\
\hline \multicolumn{4}{|l|}{ Nivel socioeconómico ${ }^{d}$} \\
\hline Alto & 9,2 & $0,66(0,44$ a 0,99$)$ & $0,72(0,43$ a 1,21$)$ \\
\hline Medio & 8,0 & $0,40(0,26$ a 0,61$)$ & $0,40(0,26$ a 0,63$)$ \\
\hline Bajo & 14,5 & 1 & 1 \\
\hline \multicolumn{4}{|l|}{ Paridad } \\
\hline 4 o más & 16,6 & $3,43(1,81$ a 6,51$)$ & $1,93(0,74$ a 5,03$)$ \\
\hline De 1 a 3 & 10,8 & $1,97(1,06$ a 3,68$)$ & $1,53(0,57$ a 4,10$)$ \\
\hline 0 & 5,0 & 1 & 1 \\
\hline
\end{tabular}

mujeres actualmente mayores practicaron menos deportes a lo largo de toda su vida) o a que en estas mujeres la práctica de deportes ha disminuido con el tiempo. Lamentablemente, el diseño transversal de este estudio no permite poner a prueba estas hipótesis y esto deberá explorarse en investigaciones futuras.

Llama la atención que las mujeres con más escolaridad indicaron que practicaban deportes más tiempo, aun después de ajustar los resultados según las otras características demográficas, como edad, región del país y área de residencia, estado marital, nivel socioeconómico y paridad. Esta asociación puede responder a que las mujeres con mayor escolaridad conocen mejor los efectos beneficiosos que para la salud tiene la práctica de deportes y ejercicios físicos, y a que cuentan también con más recursos para practicarlos. Por otro lado, el hecho de que las mujeres con un mayor número de partos informen que practican menos deportes puede ser el resultado de que estas mujeres enfrentan otras demandas en su vida cotidiana que dificultan la práctica de deportes o ejercicios. Asimismo, es interesante que la paridad se asocie con una menor actividad física entre las mujeres que viven en áreas urbanas, pero que esa relación no sea significativa entre las mujeres de áreas rurales. Los programas o intervenciones para la promoción de la actividad física deben tomar en cuenta estos resultados y proporcionar mayores oportunidades para la práctica deportiva a las mujeres con un mayor número de hijos y menor escolaridad.
Los resultados del estudio muestran que, dada la escasa práctica de deportes y ejercicios, este tipo de actividad contribuye muy poco a la actividad física total de las mujeres en México. Aunque las mujeres mayores de 30 años presentan menos posibilidades de estar en el $10 \%$ de menor actividad y más posibilidades de estar en el 10\% de mayor actividad que las de 20 años o menos, esto no se debe a la práctica de los deportes, pues las mujeres mayores presentan valores altos en su puntaje de actividad en MET, pero practican menos deporte.

El presente estudio tiene algunas limitaciones. Se trata de un estudio transversal, por lo que no es posible inferir causalidad y únicamente se pueden identificar asociaciones entre algunas características sociodemográficas 
de las mujeres estudiadas y la práctica de deportes y la actividad física. De cualquier forma, esta información es valiosa porque ha permitido identificar grupos con una mayor o menor tendencia a practicar deportes y a realizar actividades físicas, lo que ayudará a diseñar intervenciones específicas.

Si bien la práctica del deporte informada en esta investigación tiene una correlación significativa con el consumo máximo de oxígeno, no es posible cuantificar con precisión el gasto total de energía de las mujeres encuestadas, puesto que el cuestionario utilizado no estaba dirigido a determinar el gasto total de energía, sino a identificar a las mujeres con mayor o con menor nivel de actividad física. Por esta razón el análisis de este estudio se ha basado en el 10\% superior y el $10 \%$ inferior y se han agrupado algunas variables en solo dos categorías. Esta estrategia analítica, en la que se clasifica a las mujeres encuestadas en grupos de $10 \%$ según su actividad y se centra el análisis en los dos grupos extremos, hace que los errores de cálculo sean aditivos y se propaguen a los niveles intermedios de actividad física. En futuras investigaciones se recomienda estudiar los grupos de mujeres de menor actividad, quienes corren un mayor riesgo de sufrir enfermedades crónicas. Este grupo debe ser blanco de intervenciones que promuevan la actividad física.

Es importante señalar que algunas actividades moderadas, como caminar, pueden contribuir considerablemente al gasto total de energía y pueden desempeñar un papel importante en la prevención de enfermedades crónicas. En este estudio la atención se ha centrado en los factores asociados con la práctica de deportes y ejercicios como formas de actividad física pesada, porque la medición de la actividad física tiene mayor validez cuando se trata de actividades pesadas, especialmente de carácter deportivo. En el futuro se recomienda analizar la contribución de las actividades físicas moderadas a la prevención de enfermedades crónicas.

Podría argumentarse que las mujeres que dedican poco tiempo a practicar deportes pudieran haberse negado a contestar el cuestionario, por lo que los datos recogidos podrían estar sobreestimando la práctica de deportes y de actividades físicas. Sin embargo, este posible sesgo tendría poco impacto en los resultados debido al alto porcentaje de respuesta a la encuesta y a que las características de las mujeres que no brindaron la información completa sobre su actividad física no difirieron de las de las mujeres que proporcionaron toda la información.
Por último, la información contenida en la ENN-99 no permite analizar algunos factores que determinan la práctica de deportes, como los conocimientos, las actitudes y los componentes culturales. Estos problemas, así como la asociación entre la actividad física y la salud, deberán ser abordados en futuros estudios.

\section{CONCLUSIONES}

La información obtenida documenta el poco tiempo dedicado a la práctica de deportes en mujeres en edad reproductiva en México, especialmente entre las mayores de 21 años y las de bajo nivel de escolaridad, lo que a su vez puede contribuir al aumento de la prevalencia de la obesidad y de las enfermedades crónicas. Aunque se han realizado esfuerzos para estimular la práctica de los deportes en la población mexicana, es imperativo intensificar los recursos en esta área para reducir el efecto de las enfermedades crónicas en el futuro.

Agradecimientos. Los autores agradecen la colaboración de la maestra Guadalupe Rodríguez en la elaboración del cuestionario de actividad física empleado en este estudio.

\section{REFERENCIAS}

1. Consejo Nacional de Población. La situación demográfica de México 2000. México, D.F.: Consejo Nacional de Población; 2001.

2. México, Secretaría de Salud. Mortalidad 19931998. México, D.F.: Dirección General de Estadística e Informática, SSA; 1994-1999.

3. México, Secretaría de Salud. Programa Nacional de Salud 2001-2006. México, D.F.: Secretaría de Salud; 2002

4. México, Secretaría de Salud. Encuesta Nacional de Enfermedades Crónicas. México, D.F.: Instituto Nacional de la Nutrición "Salvador Zubirán", Secretaría de Salud; 1993.

5. Rivera J, Shamah T, Villalpando S, González de Cossío T, Hernández B, Sepúlveda J. Encuesta Nacional de Nutrición 1999. Estado nu- tricio de niños y mujeres en México. Cuernavaca, Morelos, México: Instituto Nacional de Salud Pública; 2001.

6. Willett W. Nutritional epidemiology. 2. ${ }^{\mathrm{a}}$ ed. New York: Oxford University Press; 1998.

7. U.S. Department of Health and Human Services. Physical activity and health: a report of the surgeon general. Atlanta: U.S. Department of Health and Human Services, Centers for Disease Control and Prevention, National Center for Chronic Disease Prevention and Health Promotion; 1996.

8. Hernández B, Gortmaker SL, Colditz GA, Peterson KE, Laird NM, Parra-Cabrera S. Association of obesity with physical activity, television programs and other forms of video viewing among children in México City. Int J Obes 1999;23:845-854.

9. Organización Panamericana de la Salud. La inactividad física: un factor principal de riesgo para la salud en las Américas. Washington, D.C.: OPS; 2002. (Hoja informativa No. 3).

10. Buchner D, Miles R. Seeking a contemporary understanding of factors that influence physical activity. Am J Prev Med 2002;23:3-4.

11. U.S. Department of Agriculture, U.S. Department of Health and Human Services. Nutrition and your health: dietary guidelines for Americans 2000. 5th ed. Washington, D.C.: Home and Garden Bulletin 232; 2000.

12. Toussaint G. Patrones de dieta y actividad física en la patogénesis de la obesidad en el es- 
colar urbano. Bol Med Hosp Infant Mex 2000; 57(11):650-661.

13. Instituto Nacional de Estadística, Geografía e Informática. Marco muestral nacional de hogares 1995. Aguascalientes, México: INEGI; 1995.

14. Bouchard C, Tremblay A, LeBlanc C, Lortie G, Savard R, Theriault G. A method to assess energy expenditure in children and adults. Am J Clin Nutr 1983;37:461-467.

15. Ainsworth BE, Haskell WL, Whitt MC, Irwin ML, Swartz AM, Strath SJ, et al. Compendium of physical activities: an update of activity codes and MET intensities. Med Sci Sports Exercise 2000;32(Suppl):S498-S516.

16. Bronfman M, Guiscafré $\mathrm{H}$, Castro V, Castro R, Gutiérrez G. La metodología y análisis de las características socioeconómicas de la muestra. Arch Invest Med 1988;19:351-360.

17. StataCorp. Stata Statistical software: release 7.0. College Station, Texas: Stata Corporation; 2002.

18. Zhang JMB, Yu KF. What's the relative risk?: a method of correcting the odds ratio in cohort studies of common outcomes. JAMA 1998;280(19):1690-1691.
19. Manson JE, Lee IM. Exercise for women: how much pain for optimal gain? N Engl J Med 1996;334:1325-1327.

Manuscrito recibido el 2 de enero de 2003. Aceptado para publicación, tras revisión, el 31 de julio de 2003.
ABSTRACT Factors associated with
physical activity among
Mexican women of
childbearing age

Objective. To document the practice of light and heavy physical activities, especially sports and exercise, among women from 12 to 49 years old in Mexico, and to evaluate the association that that has with some sociodemographic characteristics.

Methods. A sample of 2367 women 12 to 49 years old living in Mexico in 1999 was studied through secondary analysis of data from Mexico's 1999 National Nutrition Survey (Encuesta Nacional de Nutrición). The Survey used a clustered stratified multistage sampling scheme, with a final sample of 21000 homes in the entire country. A questionnaire was used to evaluate the time that the women spent on various forms of physical activity, with the activity measured on a scoring scale in terms of metabolic equivalents (METs). The dependent variables in the study were the time spent on heavy physical activities (those that require an energy expenditure of at least 5 METs/hour) and the time devoted to light or sedentary activities (such as sitting while on the job or elsewhere; watching television, a video, a movie, or a theater presentation; or sleeping). The independent variables were age, schooling, parity, region of the country, area of residence (urban or rural), socioeconomic level (measured through an index prepared based on housing conditions and household possessions), and marital status. The association between the independent variables and practicing sports was evaluated, and gross odds ratios (ORs) were calculated, with $95 \%$ confidence intervals (CIs). In order to identify the association between practicing sports and the independent variables a logistic regression model was used, adjusted for the effects of possible confounding variables. Adjustments were also made in the probability of a woman's being selected for the sample, using a weighting factor. In addition, adjustments were made to control for the design effect produced by the grouping of the observations in the sample, using the "svy" complex-samples routine of the Stata version 7.0 statistical analysis computer software.

Results. The 2367 women whose information was analyzed spent an average of 0.08 hours per day practicing sports and 1.25 hours per day on heavy physical activities. Only $16 \%$ of the women reported that they regularly practiced some sport. After adjusting for all the independent variables, the women older than 30 years had a lower OR of practicing sports in comparison to women who were 20 years of age or less (OR $=0.53 ; 95 \%$ CI: 0.33 to 0.84 ), as was also true for women $21-30$ years old $(\mathrm{OR}=0.35 ; 95 \% \mathrm{CI}: 0.23$ to 0.54$)$. Having the highest level of schooling and not having had children were both directly associated with practicing a sport. While the area of residence (urban or rural), marital status, and socioeconomic level showed differences among the groups, such differences were not significant after adjusting for the other study variables. In terms of physical activity measured in METs, the time spent on sports made up $10 \%$ of the total for heavy activity and $0.7 \%$ of the overall total. In terms of the possibility of being among the $10 \%$ of the women who expended the fewest METs per day, that was more likely for women under 21 years of age than it was for women 31 or older, and it was also more likely for women who had a primary level of schooling than for women with a secondary education.

Conclusions. This study showed that practicing sports is not a frequent form of physical activity among women of childbearing age in Mexico, especially those who are over 20 years of age and those with a low level of schooling. This situation could contribute to increases in the prevalence of obesity and chronic diseases. 